PACS: 46.25-y

UDC: $530.1 / 539.8$

\title{
Topological aspects of linear elastisity theory (methodological notes)
}

\author{
V. D. Natsyk ${ }^{1,2}$, I. M. Pakhomova ${ }^{1}$ \\ 1. V. N. Karazin Kharkiv National University, sq. Svoboda 4, Kharkiv 61077, Ukraine \\ 2. B.Verkin Institute for Low Temperature Physics and Engineering of the National Academy of Sciences of Ukraine, \\ 47 Nauky Ave., Kharkiv, 61103, Ukraine
}

ORCID: 0000-0001-9107-474x

DOI:10.26565/2222-5617-2018-28-01

A comparative discussion is given of the deformation properties of three-dimensional (3D) and two-dimensional (2D) solids, which are considered in the approximation of continuum mechanics as elastic continua with three and two spatial dimensions. Attention is drawn to the effectiveness of attracting concepts and methods of geometry to establish the general patterns of deformation of such systems without taking into account the physicochemical properties of atoms and the interatomic forces. In a geometric description, these continua are elastic spaces with different topological properties, which leads to significant differences in the relationships between the characteristics of their elasticity: Young's moduli, shear, bulk moduli, and Poisson's ratio. Deformation characteristics that can be considered as unique topological invariants of 3D and 2D elastic continua are established.

Keywords: topology, Young's moduli, shear, bulk moduli, and Poisson's ratio.

Проведено порівняльне обговорення деформаційних властивостей тривимірних (3D) i двовимірних (2D) твердих тіл, які розглядаються в наближенні механіки суцільного середовища як пружні континуум з трьома і двома просторовими вимірами. Звернуто увагу на ефективність застосування понять і методів геометрії для встановлення загальних закономірностей деформування таких систем без урахування фізико-хімічних властивостей атомів і сил міжатомної взаємодії в них. При геометричному описі ці континууми уявляються як пружні простори 3 різними топологічними властивостями, що призводить до істотних відмінностей співвідношень, що пов'язують між собою характеристики пружності: модулі Юнга, зсуву, всебічного стискання і коефіцієнти Пуассона. Встановлено деформаційні характеристики, які можна розглядати як своєрідні топологічні інваріанти 3D i 2D пружних континуумів.

Ключові слова: топологія, модуль Юнга, модуль зсуву, коефіцієнт Пуассона, модуль всебічного стиску.

Dedicating present article to memory of Yakov Evseevich Geguzin we recall one of the remarkable personality namely his gift of teacher and popularization of Physics. At his lectures and popular books scientific severity and accuracy are wonderfully and in proportion combined with the original nonstandard and often unexpected point of view about discussion subject. At proposed methodical notes we try as far as possible to follow this stylistic approach at discussion of a certain problem of modern linear elasticity theory.

\section{Introduction}

The mechanics of reversible deformation of solid states, regarded as an elastic continuous medium (continuum) draws up the content of the linear elasticity theory. Its basic equations and relations were established by Cauchy and Poisson in the second decade of XIX century [1].

Their research at that time played a very significant role in creating the prerequisites for the development and deepening of several areas and sections of fundamental mathematics, including geometry. The modern historian of mathematics notes that "in the future many outstanding mathematicians were constantly interested in the theory of elasticity with its clearly expressed geometric character" [2].

(C) Natsyk V. D., Pakhomova I. M., 2018
At present a large number of crystalline and vitreous (glassy) systems where the centers of microscopic structural elements (atoms or molecules) are located in the plane are intensively studied in solid state physics. The most general rules for geometric classification of mathematical and physical objects are developed in one of the sections of geometry - topology [3,4]. In the frame of topological representations (Appendix), a plane may be considered as a continuously filled space with two dimensions: in it the positions of the centers of elementary structural units of a solid state are determined by specifying two coordinates. Therefore, the abovementioned physical systems have been called twodimensional (2D) crystals or glasses: they have different translationally symmetric (crystals) or chaotic (glass) arrangement of elementary structural units. The study of 
two-dimensional systems has a rather prolonged history [5-7], but over the last decade interest in this problem has increased significantly due to discover and initiation of an intensive investigation of 2D graphene crystals - a plane translationally ordered system of carbon atoms [8].

Mathematical description and theoretical analysis of the properties of such $2 \mathrm{D}$ structures require a significant modification of models and methods which were developed before for 3D solid bodies: in topology, they are associated with a three-dimensional space in which the position of the elementary structural units is determined by specifying three coordinates. In particular at description the mechanical properties of $2 \mathrm{D}$ structures in the continuum approximation by methods of linear elasticity theory, it is necessary to formulate statements and equations of $2 \mathrm{D}$ elasticity theory $[9,10]$ : because the theory has specific special features and differences in comparison with the well-known elasticity theory for $3 \mathrm{D}$ solids [1]. However, some of these features are not directly related to the physics of interatomic interactions in $3 \mathrm{D}$ or $2 \mathrm{D}$ systems, but are caused by significant differences in concepts, theorems, equations and analysis methods in those sections of mathematics, in particular at geometry, which are attracted for comparative description of both clearly mathematical and physical objects with a different number of space dimensions.

The main intention of this publication is to attract the attention of teachers and students who specialize in solidstate physics and crystal physics to the effectiveness of attracting concepts and methods of geometry to describe the general laws of deformation of elastic solid-state systems without taking into account the physicochemical properties of atoms and the interatomic forces. It is also important to emphasize the specific topological features and differences resulting in the comparative description of the elastic deformations of three-dimensional and twodimensional solids. These remarks explain the expediency of using the terms "topological aspects of the linear elasticity theory".

The object of analysis and description in the article will be small deformations of isotropic solids, considered in the continuum approximation (as continuous media). It allows to use the concepts, relationships and equations of the linear elasticity theory of three-dimensional [1] or two-dimensional [9] continua.

\section{Physical space in the linear elasticity theory}

A brief description of the algorithm for "geometrization" of physical systems is given in the Appendix. Here we will consider a concrete example of such a system - solids whose atomic structure has a short (chemical) order: they consist of a large (in the limit infinite) number of basic (chemically identical) atomic groups, and the centers of the groups are located in empirical 3D space (in terms of mathematics this $G_{3}$ space with zero curvature). The translationally symmetric or chaotic arrangement of the centers, the distribution of sizes and orientations of these groups when placed in space corresponds to a crystalline or vitreous structure of solids. Neglecting the dimensions but preserving the physical properties of such groups turns them into physical points, and the continuous filling of these points with a $3 \mathrm{D}$ space or $2 \mathrm{D}$ space $\left(G_{2}\right.$ space with zero curvature) leads to the concepts of $3 \mathrm{D}$ or $2 \mathrm{D}$ material continua.

Let us note some of the most important physical properties of these continua:

- the presence in them of the force interaction between the points, which in the continuum limit corresponds to the interaction between the atoms of the basic groups, it can be divided into a contact interaction between the nearest groups (short-range interaction) and interaction at distances exceeding the interatomic (long-range) interaction;

- the ability of continuum to deform without disruption of continuity, in which individual elements of the continuum under the action of neighboring elements or external force fields can change their shape and dimensions but the continuity of point distribution is not disrupted;

- 3D volume and 2D plane solids can be detected from an infinitely extended continuum as fragments. They have sharply outlined outer boundaries that are continuously filled with points inside the boundaries and with complete absence of points outside them;

- an infinitely extended continuum, its individual regions or bodies in the initial state (before deformation) are in a state of thermodynamic equilibrium at some given temperature and keep stability with respect to arbitrary deformations.

To avoid misunderstandings it is necessary to note that the physical continuum with the abovementioned properties is the ultimate ideal model of solids, this model is applicable for describing the mechanical properties of real materials at sufficiently low values of relative deformations, until the elasticity or fracture limits are reached $[1,9]$.

To formulate the foundations of the linear elasticity theory for description the deformation properties of the 3D continuum [1], we first of all consider the simplest forms of deformation - homogeneous tension (or compression) and pure homogeneous shearing in an isotropic continuum. The study of several simple problems of this type makes it possible to introduce the concepts of moduli or coefficients of elasticity which

Вісник ХНУ, серія «Фізика», вип. 28, 2018 
serve as the physical characteristics of the continuum, establish the relationships between them and find intervals of possible change of their significances, which are compatible with the assumption of the thermodynamic stability of the continuum at the initial (not deformational) state. The same approach has been also realized in the formulation of the foundations of the linear elasticity theory for the 2D continuum [9, 10]. The comparison of the deformation properties and the elasticity characteristics of 3D and 2D continua was carried out at [10] and showed significant differences that are not related to the nature of the force interaction between physical points (between atoms in real bodies) but caused by differences in topological properties of these continua. In the following sections of the article, the differences in the deformation properties of elastic bodies with threedimensional and two-dimensional geometry will be discussed in more detail.

\section{Homogeneous deformations and elastic moduli of an isotropic 3D continuum [1]}

Let us consider a homogeneous isotropic and infinitely extended elastic medium (continuum), which at the initial state is a thermodynamic equilibrium physical system with $G_{3}$ (or $3 \mathrm{D}$ ) geometric properties of the space. The position of every point in such a space is determined by the three-component radius vector $\vec{r}=\left(x_{1}, x_{2}, x_{3}\right)=\left(x_{i}\right)$ as regards to $O x_{i}(i=1,2,3)$ rectangular coordinate system with origin at an arbitrary point $O$. The configuration of the points of medium under deformation can be described by a three-component vector displacement field $\vec{u}(\vec{r})=\left(u_{i}\right)$, components $u_{i}(\vec{r})$ and their first derivatives $\nabla_{k} u_{i}(\vec{r})=\frac{\partial}{\partial x_{k}} u_{i}(\vec{r}) \quad$ are considered as continuous functions of $\vec{r}=\left(x_{i}\right)$. In the linear theory of elasticity, the local deformations of the continuum can also be described by the tensor field of relative deformations $\varepsilon_{i k}(\vec{r})=\varepsilon_{k i}(\vec{r})$ :

$$
\begin{gathered}
\varepsilon_{i k}=\frac{1}{2}\left(\nabla_{i} u_{k}+\nabla_{k} u_{i}\right), \\
\nabla_{i}=\frac{\partial}{\partial x_{i}}, \quad i, k=1,2,3
\end{gathered}
$$

This tensor has nine components, six of them are independent $\varepsilon_{q}(\vec{r})(q=1,2, \ldots, 6)$ :

$$
\begin{gathered}
\varepsilon_{1}=\varepsilon_{11}, \quad \varepsilon_{2}=\varepsilon_{22}, \quad \varepsilon_{3}=\varepsilon_{33}, \\
\varepsilon_{4}=\varepsilon_{23}=\varepsilon_{32}, \quad \varepsilon_{5}=\varepsilon_{13}=\varepsilon_{31}, \quad \varepsilon_{6}=\varepsilon_{12}=\varepsilon_{21} .
\end{gathered}
$$

The diagonal components of the strain tensor $\varepsilon_{11}, \varepsilon_{22}$, $\varepsilon_{33}$, determine the relative change in the length of a small rectilinear segment in a continuum oriented before a deformation along one of the coordinate axes $O x_{i}$ (stretching-compression deformation), and the sum

$$
\varepsilon_{n n}=\varepsilon_{11}+\varepsilon_{22}+\varepsilon_{33}
$$

describes the relative change of the volume of a small element of the continuum (dilatation). The rule of summation over repeated coordinate indexes is used for writing of the vector and tensor relations.

Nondiagonal components of the tensor $\varepsilon_{i k}$ at $i \neq k$ determine the change of angle between two mutually perpendicular segments along the axes $O x_{i}$ and $O x_{k}$ (shear deformation).

Any local deformation of the continuum can be represented by the sum of the dilatation (the spherical component) $\varepsilon_{n n}$ and pure shear $\widetilde{\varepsilon}_{i k}$, if the identity is used

$$
\varepsilon_{i k} \equiv \frac{1}{3} \varepsilon_{n n} \delta_{i k}+\tilde{\varepsilon}_{i k}, \quad \tilde{\varepsilon}_{i k}=\varepsilon_{i k}-\frac{1}{3} \varepsilon_{n n} \delta_{i k},
$$

where $\delta_{i k}-$ Kronecker symbol $\left(\delta_{11}=\delta_{22}=\delta_{33}=1\right.$, $\left.\delta_{12}=\delta_{21}=\delta_{13}=\delta_{31}=\delta_{23}=\delta_{32}=0\right)$, at $\delta_{n n}=3$. Tensor $\tilde{\varepsilon}_{i k}$ is called the deviator of the strain tensor, of its offdiagonal components $i \neq k$ only three remain independent $\varepsilon_{4}, \varepsilon_{5}$ and $\varepsilon_{6}$ but $\tilde{\varepsilon}_{n n}=0$.

In the linear elasticity theory the bulk density of the free energy of the deformed continuum is considered as a function of temperature $T$ and six independent components of the strain tensor $\varepsilon_{i k}=\left(\varepsilon_{q}\right)$. The deformation component of the free energy of an isotropic continuum can be represented in the form of two equivalent quadratic forms:

$$
\begin{aligned}
& F\left(T, \varepsilon_{1} \varepsilon_{2}, \ldots \varepsilon_{6}\right)-F_{0}(T)=\frac{1}{2} \lambda(T) \varepsilon_{n n}^{2}+\mu(T) \varepsilon_{i k} \varepsilon_{i k}= \\
& =\frac{1}{2} K(T) \varepsilon_{n n}^{2}+\mu(T) \tilde{\varepsilon}_{i k} \tilde{\varepsilon}_{i k}
\end{aligned}
$$

Here $F_{0}(T)-$ is equilibrium value of the free energy density of an undeformed continuum, and $\lambda(T), \mu(T)$ and $K(T)$ - are equilibrium isothermal parameters that characterize the continuum's ability to accumulate elastic energy under deformations.

In the elasticity theory, the important role is played by the concept of internal mechanical stresses, which characterize the changes in the forces of short-range interaction between neighboring elements of the continuum under its deformations. To describe these forces, we introduce the tensor field of internal stresses $\sigma_{i k}(\vec{r})=\sigma_{k i}(\vec{r})$, which under isothermal reversible deformations is determined by the relations

$$
\sigma_{i k}=\left(\frac{\partial F}{\partial \varepsilon_{i k}}\right)_{T}
$$


A separate component of the stress tensor has a physical dimension $\sigma_{i k}\left[\frac{\text { force }}{\text { area }}\right]$. It means the force that is directed along the axis $O x_{i}$ and acts on a unit area with the normal along $O x_{k}$ on the interface between adjacent elements of the continuum.

Using (6) and quadratic forms (5) we obtain two types of relations between the components of the stress tensor $\sigma_{i k}(\vec{r})$ and the strain tensor $\varepsilon_{i k}(\vec{r})$ :

$$
\begin{gathered}
\sigma_{i k}=\lambda \varepsilon_{n n} \delta_{i k}+2 \mu \varepsilon_{i k}, \\
\sigma_{i k}=K \varepsilon_{n n} \delta_{i k}+2 \mu \tilde{\varepsilon}_{i k} .
\end{gathered}
$$

These are two forms of recording the Hooke's law, which determines the linear relationship of local internal stresses in the continuum with its local deformations, and the parameters $\lambda, \mu$ and $K$ have been called isothermal elastic moduli. These moduli are considered as equilibrium characteristics of the deformation properties of the continuum. Since according to (4) any deformation can be represented by the sum of the dilation $\varepsilon_{n n}$ and pure shear $\tilde{\varepsilon}_{i k}$, it is sufficient to specify only two parameters for a complete characterization of the deformation properties of an isotropic elastic continuum, for example $K$ and $\mu$ or $\lambda$ and $\mu$. Physical dimension of the elasticity moduli of the 3D continuum $\lambda, \mu, K\left[\frac{\text { force }}{\text { area }}\right]$ coincides with the dimension of the components of the tensor $\sigma_{i k}$.

Symmetric stress tensor $\sigma_{i k}$, and tensor $\varepsilon_{i k}$, can be represented by the sum of the spherical $\sigma_{n n}$ and deviatoric $\tilde{\sigma}_{i k}$ components according to the identity:

$$
\sigma_{i k} \equiv \frac{1}{3} \sigma_{n n} \delta_{i k}+\tilde{\sigma}_{i k}, \quad \tilde{\sigma}_{i k}=\sigma_{i k}-\frac{1}{3} \sigma_{n n} \delta_{i k},
$$

Then the following relations hold:

$$
\sigma_{n n}=3 K \varepsilon_{n n}, \quad \tilde{\sigma}_{i k}=2 \mu \tilde{\varepsilon}_{i k}, \quad i \neq k .
$$

The first one determines the change in the relative volume of the small element of the continuum $\varepsilon_{n n}$ under the action of the forces of hydrostatic compression $\sigma_{n n}$. Therefore the parameter $K$ is called the bulk modulus. The second one relates the pure shear strains $\widetilde{\varepsilon}_{i k}$ and the shear forces $\tilde{\sigma}_{i k}$, and parameter $\mu$ is called shear modulus. These moduli are used when writing Hooke's law in the form of relation (8). If Hooke's law is represented by the relation (7) then the parameters $\lambda$ and $\mu$ are called Lame coefficients. The comparison (7) and (8) gives the relation

$$
K=\lambda+\frac{2}{3} \mu \text {. }
$$

At the applied parts of the elasticity theory such as engineering and construction mechanics along with the shear modulus and bulk modulus, two other characteristics of the elastic properties of materials are used Young's modulus and Poisson's ratio. They are convenient for description of the elastic bodies deformation of limited dimensions, for example, tension (or compression) of a rod like a fragment of an elastic continuum of cylinder shape. A homogeneous deformation under the action of forces is considered. The force are applied to the ends of the rod and directed along its longitudinal axis with a free lateral surface. In a system of rectangular coordinates with an axis $O x_{3}$ along the longitudinal axis of the rod, the deforming stress in its volume has one component $\sigma_{33}(\vec{r})=P=$ const, created by the stress $P$ at the ends. It follows from the relations (7) (8) and (10) that under such a load a uniform deformed state appears in the rod $\varepsilon_{i k}(\vec{r})=$ const with three non-zero diagonal components of the strain tensor $\varepsilon_{11}, \varepsilon_{22}$ и $\varepsilon_{33}$. All of them are proportional to the load $\mathrm{P}$, and their relationship to each other can be represented in the form:

$$
\sigma_{33}=E \varepsilon_{33}, \quad \varepsilon_{11}=\varepsilon_{22}=-v \varepsilon_{33}
$$

Here the role of the elasticity characteristics of a solid body instead of moduli $\mu$ and $K$ is played by the parameters $E$ - Young's modulus and $v$ - Poisson's ratio:

$$
E=\frac{9 K \mu}{2 K+\mu}, \quad v=\frac{3 K-2 \mu}{2(3 K+\mu)} .
$$

Thus, the deformation properties of any isotropic 3D continuum can be characterized by a set of five parameters $\lambda, \mu, v, K$ and $E$. But since they are related by (11) and (13), only two of them should be regarded as independent and the choice of such a pair is determined by the specific conditions of the problem under consideration.

\section{Homogeneous deformations and elastic moduli of the $2 D$ continuum $[9,10]$}

Two-dimensional system of rectangular coordinates $x_{1} \mathrm{O} x_{2}$ will be used at the description of deformation of thermodynamical equilibrium homogeneous continuum with the geometrical properties of $G_{2}$ space (or 2D). The position of an individual point is defined by a twodimensional radius vector $\vec{r}=\left(x_{1}, x_{2}\right)=\left(x_{i}\right)$, where $i=1,2$, and the configuration of the deformed continuum will be described by a two-dimensional displacement field of points in its plane $\vec{u}(\vec{r})=\left(u_{1}, u_{2}\right)=\left(u_{i}\right)$ or fourcomponent tensor field of deformations $\varepsilon_{i k}(\vec{r})=\varepsilon_{k i}(\vec{r})$ :

$$
\begin{gathered}
\varepsilon_{i k}=\frac{1}{2}\left(\nabla_{i} u_{k}+\nabla_{k} u_{i}\right), \\
\nabla_{i}=\frac{\partial}{\partial x_{i}}, \quad i, k=1,2
\end{gathered}
$$


Here the components of the displacement fields $u_{i}(\vec{r})$ and the strain tensor $\varepsilon_{i k}(\vec{r})$ are considered as continuous functions of the space variables $\vec{r}=\left(x_{i}\right)$ in the plane of the continuum.

It should be noted that such a continuum has the property of an infinitely thin elastic film and its small elements can also experience a displacement $w(\vec{r})$ in the third spatial dimension, perpendicular to the plane $x_{1} \mathrm{O} x_{2}$. In the case of an inhomogeneous distribution of displacements $w(\vec{r})$ the bending deformations of the continuum are arised This is a deformation mode specific only for the $2 \mathrm{D}$ continuum, it is absent in the $3 \mathrm{D}$ continuum. We do not discuss the mode here since the main task of the article is a comparative description of the deformation properties of 3D and 2D solids. We also note that in this section we store the symbols of the previous section to denote the deformation properties and characteristics of the continuum. We emphasize only the differences in the values of the coordinate indices: here $i, k, n=1,2$ instead of $i, k, n=1,2,3$ at the preceding part. This makes it possible to display more clearly the differences of the deformation properties of solids with three-dimensional and two-dimensional geometries discussed in the article.

The four-component symmetric tensor $\varepsilon_{i k}$ (14) has three independent components $\varepsilon_{q}, q=1,2,3$ :

$$
\varepsilon_{1}=\varepsilon_{11}, \quad \varepsilon_{2}=\varepsilon_{22}, \quad \varepsilon_{3}=\varepsilon_{12}=\varepsilon_{21}
$$

The diagonal components $\varepsilon_{11}$ и $\varepsilon_{22}$ determine the relative tensile-compression strain along two coordinate axes $O x_{i}$. The sum

$$
\varepsilon_{n n}=\varepsilon_{11}+\varepsilon_{22}
$$

describes the relative change in the area of a small element in the continuum (two-dimensional dilatation). Nondiagonal components $\varepsilon_{i k}$ at $i \neq k$ determine the shear strain in the $2 \mathrm{D}$ continuum

Any local deformation in the 2D continuum can be represented as the sum of a two-dimensional dilatation (circular component) $\varepsilon_{n n}$ and pure shear $\tilde{\varepsilon}_{i k}$ :

$$
\varepsilon_{i k} \equiv \frac{1}{2} \varepsilon_{n n} \delta_{i k}+\tilde{\varepsilon}_{i k}, \quad \tilde{\varepsilon}_{i k}=\varepsilon_{i k}-\frac{1}{2} \varepsilon_{n n} \delta_{i k},
$$

where $\delta_{i k}-$ two-dimensional Kronecker symbol ( $\left.\delta_{11}=\delta_{22}=1, \delta_{12}=\delta_{21}=0\right)$, where $\delta_{n n}=2$. The deviator of the strain tensor $\tilde{\varepsilon}_{i k}$ at $i \neq k$ has only one independent component $\varepsilon_{3}=\varepsilon_{12}=\varepsilon_{21}$, a $\hat{\varepsilon}_{n n}=0$. We should pay attention to the difference in the numerical coefficients in formulas (4) and (17) (accordingly $\frac{1}{3}$ and $\frac{1}{2}$ ). Exactly this difference after all will be associated with a significant difference in the relationship between the characteristics of the elasticity of 3D and 2D continua.

In the approximations of the linear elasticity theory the two-dimensional free energy density of an isotropic deformed 2D continuum is a function of temperature $T$ and three independent components of the strain tensor $\varepsilon_{q}$ and can be represented as two equivalent quadratic forms:

$$
\begin{aligned}
& F\left(T, \varepsilon_{1}, \varepsilon_{2}, \varepsilon_{3}\right)-F_{0}(T)=\frac{1}{2} \lambda(T) \varepsilon_{n n}^{2}+\mu(T) \varepsilon_{i k} \varepsilon_{i k}= \\
& =\frac{1}{2} K(T) \varepsilon_{n n}^{2}+\mu(T) \tilde{\varepsilon}_{i k} \tilde{\varepsilon}_{i k}
\end{aligned}
$$

Here $F_{0}(T)$ - equilibrium value of the two-dimensional free energy density and $\lambda, \mu, K-$ two-dimensional analogs of elastic moduli.

The short range interaction between the points of the $2 \mathrm{D}$ continuum is characterized by a symmetric internal stress tensor $\sigma_{i k}(\vec{r})=\sigma_{k i}(\vec{r})$. Each individual component of this tensor has dimension $\sigma_{i k}\left[\frac{\text { force }}{\text { lenght }}\right]$, here force is directed along $O x_{i}$ axis and acts on the unit element of the dividing line between next regions of the continuum, which has a normal in the direction of the axis $O x_{k}$.

In the symmetrical stress tensor, we distinguish $\sigma_{i k}$ the circular $\sigma_{n n}$ and deviatoric $\hat{\sigma}_{i k}$ components:

$$
\sigma_{i k}=\frac{1}{2} \sigma_{n n} \delta_{i k}+\tilde{\sigma}_{i k}, \tilde{\sigma}_{i k}=\sigma_{i k}-\frac{1}{2} \sigma_{n n} \delta_{i k}
$$

Then, according to formulas (6) and (18), Hooke's law for a $2 \mathrm{D}$ continuum can be represented in the form of relations:

$$
\begin{gathered}
\sigma_{i k}=\lambda \varepsilon_{n n} \delta_{i k}+2 \mu \varepsilon_{i k}=K \varepsilon_{n n} \delta_{i k}+2 \mu \widetilde{\varepsilon}_{i k} \\
\sigma_{n n}=2 K \varepsilon_{n n}, \quad \tilde{\sigma}_{i k}=2 \mu \tilde{\varepsilon}_{i k}, i \neq k .
\end{gathered}
$$

The physical dimension of the moduli of elasticity of the 2D continuum $\lambda, \mu, K\left[\frac{\text { force }}{\text { lenght }}\right]$ coincides with the dimension of the components of the tensor $\sigma_{i k}$. It can be seen from the relations (21) that the relative change in the area of a small element of the $2 \mathrm{D}$ continuum $\varepsilon_{n n}$ is determined by the action of the forces of all-round tension-compression $\sigma_{n n}$. Therefore, the parameter $K$ should be regarded as a two-dimensional analog of the bulk modulus. Accordingly, the parameter $\mu$ has the meaning of a two-dimensional analog of the shear modulus, since it determines the relationship of pure shear $\tilde{\varepsilon}_{i k}$ and shear forces $\tilde{\sigma}_{i k}$ within the 2D continuum. Comparison of (20) and (21) leads to a relation between the moduli $\lambda, \mu$ and $K$ :

$$
K=\lambda+\mu
$$

At description of the deformation of plane elastic bodies of limited dimensions, it is also expedient to use two- 
dimensional analogues of the Young's modulus $E$ and Poisson's ratio $v$. Let us consider, for example, the elongation (or compression) of the $2 \mathrm{D}$ continuum strip along its longitudinal axis under the action of a twodimensional stress $P$ at its ends and in the absence of load on the side boundaries. Such a band is a two-dimensional analogue of the rod considered in the previous section. At rectangular coordinates system with an axis $\mathrm{Ox}_{2}$ along the longitudinal axis of the band, the deforming stress in it has only one nonzero component $\sigma_{22}(\vec{r})=P=$ const , created by the stress $P$ at the ends. It follows from (20) and (21) that under such a load a uniform deformed state appears in the band $\varepsilon_{i k}(\vec{r})=$ const with two nonzero components of the strain tensor $\varepsilon_{11}$ and $\varepsilon_{22}$. Each of them is proportional to the load $P$, and their relationship can be represented in the form of relationships in which the role of the elasticity parameters is played by twodimensional analogues of the Young's modulus $E$ and Poisson's ratio $v$ :

$$
\sigma_{22}=E \varepsilon_{22}, \quad \varepsilon_{11}=-v \varepsilon_{22} .
$$

The relationship between $E$ and $v$ with elasticity moduli $K$ and $\mu$ is defined by:

$$
E=\frac{4 K \mu}{K+\mu}, \quad v=\frac{K-\mu}{K+\mu} .
$$

To characterize the deformation properties of an elastic 2D continuum, five parameters can be used $\lambda, \mu, v, K$ and $E$, but since they are related by (22) and (24) only two of them should be regarded as independent.

\section{Topological differences in the deformation properties of 3D and 2D elastic solids}

If we carefully compare the basic relationships between the linear elasticity theory of 3D and 2D continuum, we can note the presence of both their similarity (at least visual), and quite significant differences. For example formulas (5) - (8) and (18) (20), visually coincide, but this similarity is conditional, it is a consequence of the use in both sections of the same symbols for marking radius-vector of physical points $\vec{r}=\left(x_{i}\right)$, of strain tensors $\varepsilon_{i k}$ and stress tensors $\sigma_{i k}$, elasticity moduli $\lambda, \mu, K, \mathrm{E}$ and Poisson's ratio $v$. Strictly speaking, there is no such similarity: first, the coordinate indices assume the values $i=1,2,3$ or $i=1,2$; secondly, the components of the stress tensor $\sigma_{i k}$ and the elastic moduli $\lambda, \mu, K, \mathrm{E}$ change their physical dimensions when the spatial dimension of the continuum changes.

Very significant differences, directly related to the general geometry (topology) of the continua. There are also clearly seen when comparing the relationships between the elasticity parameters in Section 3 and in Section 4. In order to more clearly show these differences, it is advisable to give here several relationships between the elasticity parameters of 3D and 2D solids, which are most often used when considering various specific problems of the applied elasticity theory. For elastic 3D solids the parameters $\lambda, \mu, K, E$ and $v$ are related by the relations:

$$
\begin{aligned}
& E=\frac{9 K \mu}{3 K+\mu}=3 K(1-2 v)=2 \mu(1+v), \\
& v=\frac{3 K-2 \mu}{2(3 K+\mu)}=\frac{E-2 \mu}{2 \mu}=\frac{3 K-E}{6 K},
\end{aligned}
$$

Analogues of these relations for elastic 2D solids have the form:

$$
\begin{gathered}
E=\frac{4 K \mu}{K+\mu}=2 K(1-v)=2 \mu(1+v), \\
v=\frac{K-\mu}{K+\mu}=\frac{2 \mu-E}{E}=\frac{2 K-E}{2 K},
\end{gathered}
$$

Comparison of formulas (25) - (26) with formulas (27) (28) leads to the conclusion that there are significant differences in the deformation properties of elastic continua with different numbers of spatial measurements.

Separately, we should also discuss topological differences in the allowed intervals for the change in the numerical values of the elasticity parameters. The equilibrium values of the elastic moduli $\lambda, \mu, K, E$ and $v$ for a particular solid are determined by the physicochemical characteristics of the interatomic interaction and by the intensity of the thermal motion of the atoms. At the same time, these values should be compatible with the condition of thermodynamic stability common to all solids with respect to arbitrary elastic deformations. A formal mathematical criterion of stability is the positive definiteness of the quadratic forms (5) and (18), which describe the dependence of the free energy of elastic bodies on six (2) or three (15) independent components $\varepsilon_{q}$ of the strain tensor. This criterion imposes certain restrictions on the admissible values of the moduli $\lambda, \mu$, and $K$ as the coefficients of quadratic forms. For example, such restrictions can be obtained if in the formulas (5) and (18) we consider separately two types of homogeneous deformations:

- pure dilation, when $\varepsilon_{i k}=\delta_{i k}$, where $\varepsilon=$ const

$$
\begin{aligned}
& \text { and } \widetilde{\varepsilon}=0 \text {, and } F-F_{0}=\frac{s^{2}}{2} K \cdot \varepsilon^{2}, s=2,3 ; \\
& \bullet \text { pure shear when } \quad \varepsilon_{n n}=0, \quad \text { and } \\
& F-F_{0}=\mu \tilde{\varepsilon}_{i k} \tilde{\varepsilon}_{i k}=\mu \varepsilon_{i k} \varepsilon_{i k} .
\end{aligned}
$$

From this it is clear that the condition of positive definiteness of the deformation component of the free energy $F\left(T, \varepsilon_{q}\right)-F_{0}(T) \geq 0$ is the positive values of the 
moduli $K \geq 0$ and $\mu \geq 0$. It follows from (25) and (27) that this restriction also leads to the condition $E \geq 0$ for both the three-dimensional ( $s=3$ ) and two-dimensional ( $s=2$ ) continua.

However, the ranges of values of the Poisson's ratio $v$, that satisfy the condition of thermodynamic stability for $3 \mathrm{D}$ and 2D continua are significantly different. If we take the limiting values $K=0$ and $\mu=0$ for the 3D continuum according to (26), we obtain condition

$$
-1 \leq v \leq \frac{1}{2} \text {. }
$$

and for the 2D continuum, according to (28), condition

$$
-1 \leq v \leq 1 \text {. }
$$

Thus, the range of admissible equilibrium values of the Poisson's ratio of elastic solids can be regarded as a kind of topological invariant that varies abruptly from (29) to (30) upon transition from three-dimensional to twodimensional solid systems.

The above discussion and the conclusions drawn on its basis confirm the advisability of using geometric concepts and methods in the theory of elasticity. They allow a deeper and more comprehensive description of the laws of deformation of elastic solids and reveal additional features of deformation properties.

\section{Appendix [3, 4, 11]}

For a reader who does not have a fundamental mathematical education, it is useful to explain the meaning of a number of terms and concepts of geometry that are used in the article, without excessive mathematical rigor of formulations.

Geometry, as a branch of mathematics, is based on the concepts of "point" and "space": a point is an elementary structural unit of a mathematical space $G_{S}$, that is the result of continuous and infinitely extended repetition of a point in s dimensions (or conditional "directions"): positive integer values $s=1,2 \ldots$ give the number of measurements space. It is assumed that any of the points can be considered as the origin, and the position with respect to it of any other point in space is given by a set of real numbers $\left(x_{1}, x_{2}, \ldots x_{S}\right)=\left(x_{i}\right)$ - the coordinates of the point (the symbol $i=1,2 \ldots s$ denotes a coordinate index).

In the geometric description of deformations of solids within the framework of the linear elasticity theory it suffices to consider one of the simplest types of a mathematical space - the Euclidean metric space with zero curvature. In this space we introduce the notion of the distance $l^{\alpha, \beta}$ between two points $\left(x_{i}^{\alpha}\right)$ and $\left(x_{i}^{\beta}\right)$, which is defined by

$$
l^{\alpha, \beta}=\sqrt{\left(x_{1}^{\alpha}-x_{1}^{\beta}\right)^{2}+\left(x_{2}^{\alpha}-x_{2}^{\beta}\right)^{2}+\ldots+\left(x_{S}^{\alpha}-x_{S}^{\beta}\right)^{2}} .
$$

In this case, a single point (for example, the origin) can be interpreted as a zero-dimensional space and assume that the distance of any point $\left(x_{i}^{\alpha}\right)$ from itself is equal to zero $\left(l^{\alpha, \alpha}=0\right)$.

In the early stages of the development of geometry, it was a mathematical "tool" for analyzing spatial relationships in the surrounding and intuitively perceived three-dimensional empirical space $G_{3}$ (or 3D) of the physical world. In this geometry, the topology was called its section, which dealt with a general analysis of the structure of space itself-the presence or absence of continuity discontinuities (holes or point puncturessingularity points) in it, as well as the systematization and general characteristics of the lines and surfaces defined in it with different curvatures, closed or open with the presence or absence of self-intersections etc. Later, the concepts of abstract mathematical spaces described at the beginning of this section as multi-dimensional continua of mathematical points (multidimensional numerical or even functional continua) arose and became established. By analogy with $3 \mathrm{D}$ geometry in such spaces, $G_{S}$ for $s \geq 1$ you can specify different geometric figures by a continuous repetition of points, infinitely extended in one dimension and finite in others: conditional segments, lines, surfaces, volumetric figures. The number, variety and geometric meaning of such figures are different for different values of the number of measurements $s$. In modern geometry, topology is the most general and abstract part of it, in which only properties of space and geometric objects (figures) defined in it are studied that are not related to quantitative characteristics and are preserved for all continuous space deformations and oneto-one transformations of points, invariants with respect to such transformations are systematized [3, 4, 11]. The theorem [3] is formulated and proved, according to which the main topological invariant of the space $G_{S}$ is the number of dimensions $s$; in other words, changing the dimension of space changes its topology.

Topological analysis of the most common geometric properties of spaces and geometric objects defined in them widely and effectively use various branches of mathematics [11]: symmetry analysis, vector and tensor algebra, analytic and differential geometry, mathematical field theory, theory of partial differential equations. These same branches of mathematics serve in modern physics as the basis for theoretical analysis and description of various physical systems. Moreover, for their comprehensive characterization, it also proved expedient to use geometric concepts of space and its topology [4, 12, 13]. Examples include: classification of 1D, 2D and 3D structures in solid state physics; 4D space-time 
continuum in the theory of relativity; phase space in the classical mechanics of material points; functional spaces in quantum mechanics.

The use of the geometric approach and topology methods in theoretical physics helps to describe not the details of the structure and properties of physical systems, but to establish or interpret the most general laws and relationships obtained in various concrete experiments in the study of systems of different physical nature [4, 12, 13]. An important role is played by the development of representations about the relationships and mutual correspondences between abstract spaces in mathematics and their physical analogs - different types of material continuum or physical vacuum [4]. It is possible to separate various real physical systems into extremely small subsystems (regions) and consider them as physical points. They are compared to the points of the abstract mathematical space, but they preserve and bear upon themselves a set of physical characteristics: mass, momentum, potential and kinetic energies, electric charge, and so on. Continuous and infinitely extended repetition of such points creates a physical space that is regarded as an analog of a mathematical space with its geometric and topological properties. Thus, there are prerequisites for the effective use of geometry as a mathematical "tool" in theoretical physics.

The authors are sincerely grateful to S.N. Smirnov for a meaningful and useful discussion of the article, and also want to express gratitude to T.I. Vainblat for help in translating the article.

The article is offered as an addition to the course of lectures for the masters of the Physics Faculty: Prof. Natsyk V.D. "Fundamentals of the elasticity and plasticity theory of solids", kfk.biz.ht - Educational materials.

\section{References}

1. L. D. Landau. E.M. Lifshits. Teoriya uprugosti. Nauka. M. (1978). $730 \mathrm{~s}$

2. D.Ya. Stroyk. Kratkiy ocherk istorii matematiki. Nauka. M. (1978). $335 \mathrm{~s}$

3. K. F. Kleyn. Elementarnaya matematika s tochki zreniya vysshey. Nauka. M. (1987). $431 \mathrm{~s}$.

4. V.S. Lukianets. Fiziko-matematicheskiye prostranstva i realnost. Naukova dumka. Kiyev (1971). 109 s.

5. I. F. Lyuksutov. A. G. Naumovets. V. L. Pokrovskiy. Dvumernyye kristally. Naukova dumka. Kiyev (1988). 320 s.

6. A. M. Kosevich. Teoriya kristallicheskoy reshetki (fizicheskaya mekhanika kristallov). KhGU Vishcha shkola. Kharkov (1988). 327 s.

7. A. M. Kosєvich. Mekhanika kristalichnoï gratki. seriya «Universitetski lektsiï z fiziki tverdogo tila». Nauk. vid. Akta. Kharkiv (2005). 305 s.

8. K. S. Novoselov. UFN 181. 1299 (2011).

9. V.D. Natsik. S.N. Smirnov. FNT. 39. 690 (2013).
10. V. D. Natsik. S. N. Smirnov. V. I. Belan. FNT. 44. 877 (2018).

11. G. Korn. T. Korn. Spravochnik po matematike (dlya nauchnykh rabotnikov i inzhenerov). Nauka. M. 1977. 831 s.

12. M. Nakahara. Geometry. Topology and Physics. IOP Publishing. Bristol 1990

13. A.M. Kosevich. FNT 30. 135 (2004). 